CLINICAL ETHICS

\title{
The ethics of policy writing: how should hospitals deal with moral disagreement about controversial medical practices?
}

E C Winkler

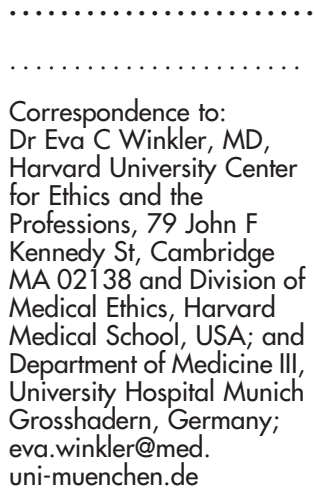

Received 26 October 2004 Accepted for publication 7 January 2005
J Med Ethics 2005;31:559-566. doi: 10.1136/jme.2004.011023

Every healthcare organisation $(\mathrm{HCO}$ ) enacts a multitude of policies, but there has been no discussion as to what procedural and substantive requirements a policy writing process should meet in order to achieve good outcomes and to possess sufficient authority for those who are asked to follow it.

Using, as an example, the controversy about patient's refusal of blood transfusions, I argue that a hospital wide policy is preferable to individual decision making, because it ensures autonomy, quality, fairness, and efficiency.

Policy writing for morally controversial medical practices needs additional justification compared to policies on standard medical practices and secures legitimate authority for $\mathrm{HCO}$ members by meeting five requirements: all parties directed by the policy are represented; the deliberative process encompasses all of the HCO's obligations; the rationales for the policy are made available; there is a mechanism for criticising, and for evaluating the policy. l $\mathrm{n}$ the last thirty years institutions have become more central to the delivery of health care than have physicians in private practice. Increasing numbers of physicians practise in large multispecialty groups and complex healthcare organisations (HCOs). As medicine becomes more specialised and its technology more expensive, these services and technologies are being merged under one roof so that they can be utilised in the most efficient way.

Institutionalising medicine means more, however, than moving doctors' offices into hospital buildings. First, the hospital has more in common with other organisations and bureaucracies than it has with the traditional doctor's office: it is hierarchically organised, has a complex division of labour, and its practices and decision making processes rely largely on rules and policies. ${ }^{1}$ Health care today is practiced by teams, not only by individual physicians. It includes health services research, mechanisms for quality review, complex networks of informational systems, logistical strategies for the coordinated use of expensive and institutionally based technologies, and it involves multiple levels of management. Health care involves several disciplines and professions beyond medicine. Physicians are but one group of professionals-albeit an essential one-who serve the goals of the healthcare organisation.

Clinical ethics is, traditionally, oriented toward the individual doctor and her patient, focusing on their rights, their actions, and how they justify their treatment decisions. Evolving as the ethics of this particular professional group, it assumes that there are two main participants only-the doctor and the patient-and that they decide jointly and in private on the course of medical treatment. Problems that arise therefore in the doctor/patient relationship are solved on a case by case basis, although occasionally with the help of an ethics consultation service.

Because of its focus on individual agency, clinical ethics fails to recognise the effects of the organisational culture in which health care is delivered, which include the effects of that culture's informal rules, and, more importantly, the effects of its beliefs and assumptions regarding ethical conduct and ethical decision making. Recent research in organisation theory and business ethics has focused on trying to understand the factors influencing ethical conduct in organisations. Issues that promote unethical behaviour are ambiguity among staff about organisational priorities, the lack of forums for discussing the ethical dimension of behaviours, and hierarchical power structures that prevent horizontal relationships. Unethical conduct can be reduced by leaders who encourage and model ethical behaviour, reward ethical conduct and discipline unethical conduct, and emphasise treating employees fairly rather than strictly obeying authority. ${ }^{23}$ Organisational ethics suggest processes that promote the organisational culture that supports ethical decision making and ethical behaviour. Less attention has been given, however, to the question: how should healthcare organisations deal with moral disagreement about medical practices?

Second, institutionalising medicine changes the setting of medical practice from private to public. The HCO is accountable for how it interacts with its physicians, patients, and multiple other stakeholders: insurers, employees, investors, and the wider community of the public. Because many people interact in a healthcare organisation and mutually influence one another and because their multiple interactions are governed by rules, the HCO is a "semipublic" place. This semipublic status bears importantly on the way the HCO should respond to moral disagreement. Unlike the individual doctor's office, where the doctor and patient may reach a private agreement within the limits of the law, the HCO must take a public stand on controversial medical practices and hotly debated ethical issues.

In the absence of a process that generates common agreement in terms of a policy, one must assume that in the HCO's view morally controversial medical decisions are best handled by the individual healthcare providers. Avoiding taking such a position and lacking an explicit policy is, in

Abbreviations: $\mathrm{HCO}$, healthcare organisation; $\mathrm{OE}$, organisational ethics 
itself, taking a stand and must be justified. Because policies guide individual action and thereby constitute the collective action of the organisation, they play an important role in modelling the interaction of the HCO with its different stakeholders. ${ }^{4}$ Therefore HCOs must carefully consider their policies and rules so as to make them justifiable. This is especially critical for policies that address controversial medical practices about which reasonable people disagree such as abortion, organ donation, or palliative sedation.

Traditional clinical ethics, with its concept of the private doctor/patient relationship, does not address the question of the HCO's semipublic role and how it should respond to moral disagreement about controversial medical practices. Therefore, political and administrative ethics, rather than merely clinical ethics, should inform our understanding of ethical problems, behaviours, and obligations in HCOs.

I shall argue that a consensus building process leading to hospital wide policies is the better alternative to individual decision making because it respects the autonomy of all parties involved and better ensures quality, fairness, and an efficient decision making process.

\section{INDIVIDUAL DECISION MAKING V A STANDING POLICY BUILT BY CONSENSUS}

Consider the following example of a morally controversial problem that has been long discussed in the medical ethics literature and that has generated considerable disagreement among reasonable people: should adolescents-most of the patients in the reported cases are Jehovah's Witnesses-be allowed to refuse blood therapy, even if this decision could result in serious injury, permanent disability, or death?

There is widespread societal consensus that adults with decision making capacity have the right to refuse blood transfusions even if it is detrimental to their health. This is grounded in respect for the patient's integrity and her right to make autonomous decisions based on religious or moral values. $^{5}$ There is also widespread consensus that parents should not refuse blood on behalf of young children, if doing so will potentially harm the child. Controversy arises, however, in the case of so called "mature minors"adolescents who, although not yet 18 years old, appear mature enough to make their own decisions. Some ethicists believe that the autonomy and integrity of mature minors should be respected, and that they should be permitted to make their own medical care decisions. ${ }^{6}$ Others believe that society's interest in protecting the health of minors should prevail, especially if a minor's decision could result in serious injury, disability, or death. Because such autonomy is not developed fully in the case of minors it is considered that this interest prevails over the concern to respect patient autonomy. ${ }^{7}$ Reflecting the range of opinions in the literature on human development, courts' assessments of the age of "maturity" have varied from 14 to 18 years. ${ }^{8}$

If this problem arises in a hospital-let us call it Catholic Hospital in Big City, Iowa-it seems compelling at first glance for the hospital to leave to the individual clinician and his patient the decision about the kind of treatment on which they both could agree. The first reason in its favour is that this approach seems best suited to preserving the autonomy of the parties involved - a central principle of clinical ethics. ${ }^{9}$ Second, case by case decision making allows the particularities of each case to be aired, and thus is more conducive to reaching an ethically appropriate solution than following a general policy. As will be seen, however, leaving the decision to the individual healthcare provider does not always result in an ideal outcome. Consider the following examples based on actual cases.

Patient $\mathrm{AB}$ is a 14 year old Jehovah's Witness, an epilepsy sufferer, and long term patient at the Catholic Hospital in Big
City, Iowa. She is scheduled for brain surgery in a week. AB rejects blood on the basis of her beliefs and expresses that verbally to her neurologist. Her parents support her decision. To prepare her for a bloodless operation her neurologist gave her erythropoietin to stimulate her blood production. The surgeon assigned to operate on her, whom she has not yet met, is aware that she is a Jehovah's Witness, but is unaware of the implications of holding that belief and the patient's powerful expression of faith. Aware that judges routinely override parental refusals to consent to blood transfusions for minor children, and because of his sense of professional integrity and his own moral values, the surgeon decides not to start with the surgery without court permission to forgo transfusion therapy.

This conflict leads to an interruption of the relationship between the patient and the hospital, and the patient reschedules her procedure with an accommodating institution. She is disappointed that her surgeon had not anticipated these problems, did not respect her personally held values, and regrets the loss of her longstanding relationship with her doctors and the Catholic Hospital.

Patient YZ, a mature minor, is admitted to the hospital for appendix surgery. The surgery has a low but present risk of clinically relevant blood loss, but the patient, a Jehovah's Witness, refuses any transfusions that might be necessary during the treatment. The patient is alert, lucid, and very adamant about his refusal of blood. Although the attending physician agrees with the patient's request that she operate without transfusion therapy, the anaesthesiologists are unwilling to administer anaesthesia under these circumstances. Having asked the hospital's risk manager for advice on how to proceed, the surgeon contacts the department of anaesthesia and finds an anaesthesiologist willing to honour the patient's wishes. The surgeon does not succeed in getting the involved parties to agree on what options should be taken if, during surgery, the patient needs blood. As a result, the issue remains unresolved, risking the hospital's fidelity to both physicians' decision not to provide transfusion therapy. In an undoubtedly positive outcome, the surgery is completed without the need for a blood transfusion.

In the case of Patient $\mathrm{AB}$ the surgeon lacked even a minimal understanding of the implications of caring for a patient who is a Jehovah's Witness; nor did his value system take into account a patient's right to refuse blood products. In the case of Patient YZ both physicians understood some of the implications of caring for a Jehovah's Witness, and both were willing to allow the patient to refuse blood. Also, the positive outcome of the surgery was purely fortuitous, obviating the need for either physician to make a decision. The number of care givers involved in any given medical practice precludes, however, there being enough time to reach a consensus on the procedural options if the patient needs blood. Both of these cases rely on assumptions that are implicit in the principle that only individual decision making can meet the needs of patients: namely, that an ideal match or consensus on shared values exists; that all parties involved have mastered the relevant knowledge, and that only the particularities of the case at hand matter in achieving the best outcome for patients.

We can argue against these assumptions in terms of the autonomy the parties retain; the fairness with which patients are treated; the efficiency by which the parties reach their decision, and the quality of the outcome reached on the basis of such decisions.

\section{The argument from autonomy}

People may hold diverse moral convictions for various reasons. They may, for instance, disagree about the facts and about the likely consequences of an action, about how 
the relevant values and conflicting principles are prioritised, or about the moral status of the entities that are central to the case. We must constantly review our interpretation of facts, the order we impose on our values and principles, and the trade offs we deem admissible, and we must do so in the light of our own experience, of new information, and of changes in our society. This kind of review in all its complexity constitutes our moral consciousness and hence is an important part of who we are. ${ }^{10}$

Those who prefer individual decision making over an explicit policy reached by consensus justify their preference on the grounds of protecting the autonomy of the parties. They assume that a standing policy interferes with the autonomous decision making of the parties. We can easily conceive of a policy that does just that-one that allows for transfusing patients against their will, or that binds healthcare providers in a way that permits patients to refuse blood against the providers' moral convictions. Such policies would, however, be deemed unethical. Both healthcare providers and patients would understandably feel their autonomy to be compromised if they were forced to act against their deeply held moral convictions.

Therefore, if respect for autonomy is to be taken seriously, all parties should be requested to consent to a morally controversial practice before being obliged to participate. Because in the hospital setting the doctor and patient are no longer the only participants, as the case of YZ shows, it will require considerable effort to bring about agreement and to reach this ambitious goal.

In today's highly specialised medicine, the decision to operate without transfusion therapy involves many parties in addition to the surgeon and her patient: the anaesthesiologists who manage the patient's vital functions; the operating room nurses who assist in preparing the patient and in performing the operation; the postoperative unit personnel who monitor the patient when she awakes from anaesthesia, and members of the surgical ward who care for her until her discharge.

If such a group of care givers do arrive at a consensus about a given case, the organisation of which they are members already has moved in the direction of developing a general rule or policy. Therefore, when juxtaposed to a procedure for reaching consensus before emergencies arise, consent to any procedure on a case by case basis seems, at best, very impractical.

Having a policy in place, in itself, does not compromise the autonomy of the parties nor does constraining the scope of discretion. In fact, autonomy can be enhanced if the policy empowers the parties to deliberate thoughtfully, rather than (when no policy exists) leaving it to them to make a sound decision under time pressure.

The belief that the doctor and the patient can make autonomous decisions in a vacuum is also misguided in what it leaves out and thereby implicitly denies-the organisational culture and unspoken traditions that govern decisions in organisations. People solve problems in organisations in the same ways they solved problems in the past. According to organisation theory, tradition and myths are part of what contributes to the so called organisational culture. ${ }^{11}{ }^{12}$ This is true for any organisation but it is especially true for healthcare organisations because the case based method is so prevalent in medicine: it is not only the way that medicine is taught, cases are also discussed and reviewed in situations such as rounds, case conferences, and mortality and morbidity conferences. How a physician handles a case is not perceived as being relevant only within the boundaries of the given case, but is interpreted within his or her tradition of similar and dissimilar cases. In that context the physician is able to draw conclusions about future cases. Therefore, it is likely that a physician's decision to comply with or to resist a patient's request to abstain from using blood transfusions is framed by similar, earlier cases. We can refer to this as "temporal framing". Over time, temporal framing may result in an informal rule that functions very like a policy, although it is one that is hidden, unspoken, and informal. The risk of having a hidden rather than an institutional policy is that it fails to function openly, and therefore its rationale (if there is one) is accessible neither for debate nor for verification.

\section{The argument from fairness}

The differential treatment of the patients $\mathrm{AB}$ and $\mathrm{YZ}$ makes clear that an absence of policy may lead to a variation in treatment not justifiable on the merits of the case. If, randomly, YZ had been assigned to the first surgeon, he probably would not have had his appendix removed; and if the second surgeon had cared for $\mathrm{AB}$ over the years, she might have been fortunate enough to have remained in his care until the end of her surgical treatment for epilepsy. The patients are similar in all relevant respects: risk of bleeding, views regarding blood transfusion, and age. Factors unrelated to these considerations, namely the value systems of their surgeons, caused the differences in their treatment. From an individual perspective, the actions of the surgeons may be morally blameless. AB's surgeon might consistently refuse to operate on patients refusing transfusion therapy, and YZ's surgeon might regularly consent to honour the wishes of such patients. From the institutional perspective, however, the failure to deal with similar cases similarly constitutes unfair treatment. When she enters the hospital the patient expects to receive the same treatment as others in her situation irrespective of the physician to whom she is assigned. It is the hospital's obligation to ensure that such consistency is guaranteed in every case and maintained over time. Moreover, without common agreement about the kinds of reasons that should count as sufficient for declining treatment the individual healthcare provider is left with the evaluation of reasons. A variety of reasons, some unrelated to the case, may come into play, ranging from serious conscientious objection to the less convincing (avoiding the emotional pain of seeing somebody die). If there is no common rule, then there is no common standard allowing for case comparison.

\section{The argument from efficiency}

Ideally, any effort to answer the question as to whether a mature minor should be permitted to refuse blood transfusions should consider all the relevant experience and knowledge that bears upon it, including the current state of the ethical and legal debate, the research in developmental psychology on the maturation process of moral judgment, and experiences with bloodless programmes in other hospitals. Each case should be evaluated against this accumulated knowledge. In the non-ideal setting of a surgeon's day, which includes preoperative rounds and his duties in the operating room, the postoperative unit, the ward, and the clinic, limited time is available for education. (In any event, the mature minor issue is so rare that few surgeons would list it as an area of research.) Thus, the time needed for the parties to consent and to form a sound opinion seems too demanding and thus not feasible. The reality often resembles the two cases described above. Therefore, a deliberative process that investigates questions in depth and provides reasons for a policy better serves efficiency than individual decision making, where neither the requirements for deliberation nor for agreement are met. The argument from efficiency claims that a standing policy obviates the need for repetitive discussions of the fundamental question, 
thereby freeing up limited decision making resources and enabling individual decision makers to focus on the particularities of the case. The policy guideline provides intermediate reasons to which one may appeal when the need for a decision arises. This intermediate level is justified by reference to the deeper concerns on which it is based; however, it is unnecessary to investigate such concerns in depth for every case. ${ }^{13}$

In the above cases, this intermediate level would be exemplified by a policy that (1) permitted minors to refuse blood transfusions where experts had determined that they were capable of making this decision, and (2) allowed individual physicians to decline to participate in such care if, and only if, such involvement would violate the clinician's deeply held moral beliefs. In other words, the underlying deeper concerns that might justify such a policy are respecting the autonomous decision of the minor, and respecting the care giver's conscientious objection.

\section{The argument from quality}

By policy mediated process, hospital and care givers can consider and form opinions on aspects of recurring situations before they materialise. This advance commitment to a series of actions not only minimises the discomfort experienced when people are pressed for a decision under uncertain conditions and time constraints, but also enables the care givers, and hence the institution, to achieve outcomes not otherwise possible. Hospitals that find this argument from quality convincing have set up so called "bloodless programmes", which include preparing the patient with erythropoietin, using operating techniques that require no blood, grouping into teams clinicians who are skilled in operating without using blood transfusions, and having a mechanism for quality control. Unless the institution has an advance commitment to a policy of reducing the need for blood, it will be unable to orchestrate all the necessary on a case by case basis.

Another reason that strengthens the argument from quality is that the course of treatment needs to be predictable. The highly specialised way in which health care is practised, divided as it is into many different tasks and carried out by different specialists, makes communication with and reliance on others to do their jobs well all the more important. If the decision making is ad hoc, exactly what will happen during treatment is difficult to predict and to explain to patients. For example, if there are unexpected complications in the course of treatment or a change in personnel, the patient may be cared for by clinicians who may not feel bound by the initial surgeon's decision, or who may even feel morally obligated to administer blood. Therefore, if clinicians assure patients that they will be treated without blood this statement might mean only that they believe that the patients will not need blood transfusions: it may not reflect their views as to what would happen if blood became necessary to save the patient's life.

Predictability about what will happen not only ensures coordination of care toward a shared aim but is also necessary to adequately inform the patient, thereby laying the foundation for an informed decision. An institution that speaks with one voice makes the patient feel safe.

I have argued that having a policy is preferable to individual decision making because it is better suited to preserving the autonomy of the parties involved; to treating like cases alike; to allocating resources in an efficient way, and to improving the result through advanced planning and coordination of care, thus enabling all parties involved to rely as fully as possible on the predictability of the process and of the outcome.

\section{WHAT POLICIES SHOULD CLINICIANS FOLLOW?}

It is of course not true that any policy is better than individual decision making. In order that a policy possess sufficient authority for those who are asked to follow it, the rule itself and the issuing organisation must meet certain conditions. These conditions differ for policies on morally controversial medical practices as opposed to policies on standard medical practices.

\section{Policies that direct standard medical practice}

At first glance the legitimacy of policies and formal rules for medical practice in a complex HCO seems to be justified on the ground that they ensure high quality and efficient care. Policies help coordinate and direct the actions and efforts of the various healthcare providers toward these shared goalsfor example, in order to prevent potentially detrimental transfusion errors, many hospitals have a rule that instructs nurses and physicians to cross check the blood group of transfusion blood with the patient's blood group at the bedside before administering the transfusion, even though the blood bank has already performed that test in its standard series of tests. This rule reflects the cumulative understanding from long experience that information can be lost or be misinterpreted each time it is transmitted from person to person. The rule thus establishes a safety mechanism that offsets the flaws created by the otherwise necessary division of labour.

The rule is over inclusive in the sense that physicians and nurses must cross check every transfusion bag for every patient so that the rare cases of mismatch are detected, but it has proved to be the best way to maximise the overall safety of transfusion treatment.

By regulating this practice and by making public its policy on this issue, the hospital indicates that patient safety is important in its institution. Although each physician acts on her own prior obligation to ensure patient safety, the fact that her actions are further informed by the hospital's policy transforms the action into one that the hospital also intends and that she performs on its behalf. ${ }^{14}$ The physician is then acting not only by virtue of being a doctor but also as a member or representative of the HCO. As a member, she must comply with its rules even if she has not independently judged the merits of performing the blood test. In many years of cross checking transfusion blood she may never have discovered a mismatch, and may therefore have concluded that the blood bank's cross check is reliable, obviating the need for the bedside test. She is not, however, at liberty to ignore the practice solely on the basis of her judgment. Why? What reasons legitimate the authoritative character of this rule? One formal reason is that by signing an employment contract with the hospital, she consented explicitly to comply with the rules and policies of the HCO. ${ }^{15}$

This merely formal justification for the authority of rules is inadequate on two counts. The first is that the physician has been hired by the HCO precisely because she contributes her professional expertise and judgment to solving medical problems. Her contract should not require her to surrender all her expert judgment on the merits of a required action. Rather, her consent to obey the rules and regulations of the hospital derives from the goal she shares with the institution: to care for patients. The contract then comprises a tacit extension: to obey the rules so long as they are not at odds with what she is hired to do, that is to provide good patient care.

The second reason why the argument from contractarian consent does not sufficiently justify requiring physicians to comply with hospital rules is that the contract cannot ask the employee to surrender his moral judgment-for example, if a rule instructs a nurse on a clinical study to draw samples of 
blood from a group of patients without their consent, the nurse can refuse to obey on moral grounds.

In short, the formal justification of authority does not give the clinician sufficient reason to obey every rule. The authoritative strength of a rule cannot be justified on grounds that are completely unrelated to the rule's content or to the legitimacy of the authority itself.

What requirement must an authority meet in order to be legitimate? One important criterion is the welfare enhancing quality of the directive, where "welfare" includes not only the beneficial outcome to the patient but also the help it affords employees in better fulfilling their obligations. The hospital's rule is justified if the judgment reflected in the rule is more likely to be welfare enhancing than the judgment of the individual subject. ${ }^{16}$ Because the hospital has a broader view of the incidents of transfusion mismatches, it is in a better position than any one individual to make a judgment on the merits of the rule concerning transfusion safety. This seems to be a sufficient reason for the doctor to comply with the rule for a bedside test even if she herself has never detected a mismatch.

Should this compliance also translate into compliance with all the other rules? Clearly, if every policy or rule is open to challenge by every employee, the advantage of the rule in providing guidance on which each employee can rely for his or her action will disappear. When an institution's rules and policies enable employees to better fulfil the obligations placed on them by their different areas of responsibility, it earns their trust in the legitimacy of its directives. Nevertheless, this should not lead to blind trust. Every clinician should test a rule roughly for obvious professional and moral errors. The professional check looks for outright mistakes in what the policies prescribe-for example, using a method that has recently been proved to have dangerous side effects-the "ethics check" looks for ethical requirements that the rule has omitted. If the rule passes these scrutinies, the healthcare provider has good reasons to accept it. Nevertheless the institution should provide a procedure that the healthcare provider can easily access to voice concerns about any rule and to learn about the rationale behind it.

Merely accepting the authority of the institution provides only a secondary reason for following its rules. The primary reasons are that the rule is welfare enhancing in both senses as noted above, and is issued by a trustworthy authority that provides a public procedure for voicing concerns about the rule and for revising it in the light of appropriate knowledge.

\section{Policies that direct morally controversial medical practices}

In contrast to the medical practice guideline, which is evidence driven and aims to ensure the standard of care, morally controversial practices do not have one single uncontroversial solution. ${ }^{17}$ The special nature of moral disagreement is that reasonable people disagree about a practice or a principle on moral grounds. Not all policies that inform ethically charged practices remain controversial. Those dealing with patient confidentiality or the use of advance directives have become so widely accepted that they have been enacted into law. Others, such as palliative sedation, which renders a terminally ill patient unconscious for the rest of her life, are still debated within the medical profession. At different levels of society, controversy about other practices, such as abortion and physician assisted suicide, still prevail. Moral dissatisfaction can also arise locally within a single HCO. Debates such as how to assist employees during downsizing, or how to allocate resources for free care, can contain diverse but valid moral reasons. Such disputes give rise to intense moral distress and should be investigated thoroughly.
Because an institution cannot rightfully claim that it alone has the cumulative expertise and administrative oversight to make the best decisions, the HCO should engage in debate about the reasons for making specific moral judgments, rather than simply stipulating what is the right thing to do.

Therefore, in articulating policies about morally controversial medical practices, further justification for these policies must supplement those that justify standard medical practices. We should move beyond what the policy ought to say to how the policy ought to be determined. The process now involves finding a reasonable answer to a given controversial question and building agreement around that solution. ${ }^{18}$ Content and procedure are equally important in resolving moral disagreement. They mutually enforce the moral soundness of the resolution of the specific moral disagreement.

\section{THE ETHICS OF POLICY WRITING FOR MORALLY CONTROVERSIAL PRACTICES}

To possess legitimate authority for governing individual action, policies on morally controversial practices must meet certain additional procedural and substantive requirements:

1. The parties that must comply with the policy must be represented.

2. There must be a deliberative process that is based on facts and that covers the scope of the HCO's obligations.

3. The rationales for the policy must be publicly known and accessible.

4. There must be mechanisms for conscientious objection and for revising the policy.

5. There must be specific procedures for ongoing evaluation of the practice.

To illustrate, I shall continue to use the hypothetical case of Catholic Hospital in Big City, Iowa, which discusses the problem of adolescents who refuse transfusion therapy and attempts to develop a consistent hospital wide approach for solving this problem. I will name the deliberative body charged with drafting the policy the organisational ethics (OE) working group.

\section{Representation}

The deliberative body appointed to develop a hospital wide policy on morally controversial practices should, first, represent all the expertise necessary for solving such problems. Because expertise is the basis on which the policy must be grounded, this first criterion is obvious.

The OE working group should represent all the parties who must implement the policy. This second criterion-participation-expresses the hospital's respect for those who will be guided and directed by the policy. It is based on a concept of the policy writing process for morally controversial practices, and more closely resembles a political process that takes various constituencies into account than it resembles authoritarian command. Giving those directed by the policy a voice in its creation implicitly recognises them as moral agents. Therefore, such a concept of participation is the appropriate response to moral disagreement in semipublic places such as an HCO.

Moreover, it is a judicious device that can elicit moral arguments, legitimate moral dissatisfaction, and become the voice of those previously excluded from such respectful participation. It can eventually accommodate opposing positions in controversies and thereby win the consent of opponents. By incorporating this concept of participation, a hospital's OE working group's policies become acceptable to all members of the institution. 
Because administrators' moral expertise is no greater than that of healthcare professionals, an authoritarian rule issued by the hospital's administrator or clinical director would lack this legitimacy. A democratic voting process need not occur for every hospital policy; rather there should be a policy writing process that includes representatives of the involved parties. A democratic voting process, impractical for many reasons, is inefficient especially given the turnover of personnel. Moreover, merely voting may not help establish the deliberative body, with its consensus building role, which is crucial for minimising disagreement.

The OE working group at Catholic Hospital therefore comprises representatives from all areas of the hospital that are involved in the treatment of patients who refuse blood therapy: surgeons; anaesthetists; nurses; legal counsel; chaplains; blood bank personnel; ethicists, and administrators. Representatives of Jehovah's Witnesses are invited to present their views, but they may not determine the final recommendation. This is justified by the distinction between being affected by and being directed by a rule. Jehovah's Witnesses ought to have their interests considered, because they are affected by the hospital's policy, but the right of policy writing is reserved for members of the institution who, because of their expertise and the responsibility implicit in it, must act in accordance with the policy.

\section{Deliberative process}

Through its deliberative process, the OE working group builds a consensus on the position a hospital should take on any morally controversial practice. The conditions facilitating this process and improving its outcome are:

(1) the comprehensiveness and quality of the working group's knowledge of the facts;

(2) working group members acknowledging that they are, morally speaking, a community of equals, and

(3) a substantive account of the obligations of a HCO.

\section{Comprehensiveness and quality of the facts}

Moral disagreement in medical matters sometimes arises from incomplete understanding of the facts or differential interpretation of the situation at hand-for example, a differential estimation of the bleeding risk and chances of survival for an individual patient if she undergoes the operation without blood transfusions. The first step in deliberating, then, should be to identify the relevant facts so that disagreement about them and their interpretation is reduced.

The OE working group at Catholic Hospital in Big City starts by reviewing their clinical cases, gathering data on how blood therapy is distributed, learning about the policies and experiences of other hospitals with "bloodless programmes," and examining discussions in the literature about ethical, legal, psychological, and developmental issues in such cases.

The second step is to evaluate the background conditions, which must include discussing the influence of hidden rules in organisational contexts- "this is how we do it here" must be fully articulated before any policy that revises "how we do things" can be adequately conceived. Those implementing the policy must understand the "policy" to which they unconsciously adhered before articulating the solution that the new policy envisions. Analysing the background condition is the foundation for implementing the policy.

To understand the existing culture at Catholic Hospital, the OE working group surveys the staffs of the intensive care unit, the department of anaesthesia, and the department of surgery. The survey reveals that only a minority of healthcare professionals are currently willing to abide by a patient's refusal of blood products. It shows that some staff object because they are unfamiliar with Jehovah's Witnesses and their beliefs; some because they lack the knowledge about the law and fear the legal consequences if their inaction allows the patient to die, and some because of their own religious beliefs that life should be protected by all means. This latter group refers to the hospital's mission statement, which emphasises the "holiness of life as a gift".

\section{Acknowledging a community of equals}

A deliberative body that is composed of members of its own institution risks simply mirroring the power structure and the interests of the institution. Such a body instead should be seen as an "ethics think tank:" a moral space for reflection in which what dominates the deliberation process is the desire to explore the issue fully and fairly rather than the strategic interests of the institution. Thus the institution's economic interests become only one of many factors the body might discuss. The OE working group does not seek consensus for its own sake but rather a consensus that is morally justified. Therefore an important ground rule for discussing the issues should be that all members of the group perceive themselves as a community of equals, morally speaking. ${ }^{19}$

\section{Substantive account for the obligations of $\mathrm{HCO}$}

Beauchamp and Childress have proposed four principles, which can help to clarify the moral values at stake in clinical ethics: respect for the patient's autonomy; non-maleficence; (primum non nocere), beneficence, and justice. ${ }^{9}$ Because there is no obvious way to prioritise these principles when they conflict, some ethicists object that these are not principles in the sense of guides for action. They do, however, represent categories of moral concern that should be considered in dealing with an ethical problem. Hospital ethics committees might indeed use these so called principles as "checklists for moral concerns... used primarily as a means of consciousness raising" ${ }^{20}$ Even thus used, the four principles seem to provide a helpful framework for clarifying treatment decisions.

My claim is that if an OE working group uses categories of moral concern as a deliberative framework, the principles of clinical ethics must be amended by the additional categories of concern that are part of the organisational setting in which the action takes place. They should reflect not only the physicians' responsibilities to their patients but the organisation's responsibilities to the entire patient population, as well as to insurers, employees, investors, and the wider community. Although the sphere of clinicians' obligations to their patients is constantly discussed, and although these guiding principles are articulated under the rubric of clinical ethics, there is no comparable set of ethical principles upon which all participants agree that guides the behaviour of HCOswhether those organisations are profit making or non-profit making.

Elsewhere I propose four principles that are proper to the four roles that HCOs are expected to fulfil: as care giver, to care with compassion; as employer, to treat employees with respect; as corporate citizen, to act in a public spirit; and as administrator of resources, to spend resources reasonably. ${ }^{21}$

According to these principles, the deliberations of the $\mathrm{OE}$ working group could be described as follows:

\section{Care with compassion}

As care giver, the HCO should seek to provide the best care possible for its patients. Although patients who refuse transfusion therapy voluntarily accept a greater risk, the hospital can minimise this risk by acquiring competence in techniques for conserving blood. Guided by this principle the hospital might set up a "bloodless programme". If its resources do not allow for that, the best alternative is to 
transfer, or to refer, the patient to a hospital that is experienced in bloodless surgery.

\section{Treat employees with respect}

As employer, the HCO should be aware of what it is asking of its healthcare providers. In the worst case scenario its care givers may have to watch a patient bleed to death without the intervention that might save the patient's life. In some cases, reluctance to provide care to patients who refuse blood may stem from a desire to avoid the emotional pain of either watching the patient die, or seeing the serious harm caused to a patient when that might have been avoided by using blood. Although in many cases such pain cannot be avoided, it may be more tolerable if staff members are supported by their colleagues and by the hospital's broader community. The hospital should allow individual physicians to decline to participate in the care of such patients out of a conscientious objection provided that the hospital does not abandon the patient. To refuse to recognise such conscientious objection should be seen as a violation of the physician's deeply held moral values.

\section{Act in a public spirit}

As a corporate citizen with a semipublic function, the HCO's decisions are evaluated with respect to the community it serves. More often than not it has a community mission and is accountable to the community for how it interacts with its patients and multiple other stakeholders. Its special responsibility is to aim for equitable access to health care for all members of the community. The Jehovah's Witness community might feel discriminated against or abandoned if the HCO's policy proposed transferring such patients to other hospitals, especially if the HCO offers specialised care that is not available elsewhere.

\section{Spend resources reasonably}

Wise stewardship of resources means using the least costly methods of achieving the ends of health care, and allocating resources fairly. A decision favouring a particular investment-for example, investing in an epilepsy monitoring unit-is, therefore, always relative to the urgent investments needed for other groups of patients.

On balance, one could conclude that the hospital should aim to set up a bloodless programme, and thereby take a stand on respecting patients' values and religious beliefs, provided that adequate resources are available. The four categories of concern or principles for organisational ethics are suggested as a framework for analysing the moral obligations of an HCO. The relative weight of a particular principle depends on the kind of question at stake and each principle needs to be defined deliberatively in relation to that issue.

The three requirements for the deliberative process set out here-to gather validated data, to strive for rational discourse, and to give a substantive account of the obligations of the institution in any given situation-are the important prerequisites for an evenhanded and sound policy.

\section{Availability of rationales}

The availability of rationales condition requires that the reasons for adopting a certain policy on morally controversial practices be made accessible to healthcare providers, current and future patients, and the public. Since HCOs are understood to be semipublic institutions, this condition provides a public account of the commitments to which the HCO adheres in making decisions on the ethical issues it debates and resolves. ${ }^{22}$ Making the policy known to staff internally is necessary in order for them to make medical decisions in accord with such policy. More importantly, giving reasons why the institution takes this particular stand in an ethically controversial debate has three desirable consequences. First, staff can share the policy with the patients, showing them that they are treated not arbitrarily but in accordance with similar cases and based on deliberation. Second, healthcare providers learn about the rights and values to which the institution is committed and can also apply them in other areas. Third, when clinicians give patients reasons instead of orders, both patients and clinicians recognise one another as moral agents, thus preventing moral reasoning from being reduced to the mere following of a rule.

Making policies and their rationales publicly accessible serves as advance notification to future patients, future staff, and the community the organisation serves. If the hospital has a "treat without blood" policy, Jehovah's Witness patients have good reason to hold this hospital accountable for treating them according to the policy. Access to policies also helps a future employee discover the institution's stand on morally controversial issues-for example, abortion or euthanasia-and whether it is at odds with his or her own deeply held moral beliefs.

\section{Exception for conscientious objection and mechanism for modifying policies}

The participatory process of policy writing cannot completely eliminate moral disagreement. The organisation can, however, further reduce the likelihood that a policy will compel care givers to act against their moral conviction by including an option that is analogous to that which patients use when they refuse treatments. This can be done by introducing into the policy an exception (conscientious objection) that allows care givers to decline to participate in a practice because of their strong moral or religious beliefs. In this respect, however, the burdens of proof are asymmetrical between the physician and the patient. The patient need not claim a reason of deep conscience: making an informed and autonomous choice is sufficient for him, even though his choice may rest on such a reason. The physician, however, must prove why she is deeply opposed to delivering such care. This asymmetry is grounded in the difference in the need for entering the relationship (the patient has no choice but to seek treatment, but the physician has a duty to treat), and in the difference in risk to one's integrity (the patient's bodily, psychological, spiritual, and moral integrity is at stake, whereas the physical integrity of the physician is not endangered). The burden of proof therefore is with the clinician who must justify her decision to decline to participate in treating a patient on the basis of valid moral reasons.

Another important condition for the legitimacy of the policy is a mechanism that makes channels for constructive criticism readily accessible. ${ }^{23}$ In the light of new evidencefor example, working experience under the given policy, or changes in societal evaluation of it-it might prove necessary to revise or adapt a policy. The opportunity for staff and patients to voice their reservations about a policy imbued practice affords an additional procedure for keeping the HCO accountable. ${ }^{24}$

\section{Implementation and evaluation}

The implementation of the policy is not a trivial last step in the deliberative process but the very essence of taking what we know about organisations seriously. It is the point at which research in organisational theory, learning behaviour, and culture is brought to bear.

Hospital ethics committees have been writing policies on important ethical questions for many years. Along with education and case consultation it is in fact one of their three main functions. ${ }^{25}$ Nevertheless it remains true that unless policies are responsive to the hospital's predominant 
background conditions they will remain unknown and no one will follow them. ${ }^{26}$ Senior leadership, especially in hierarchical organisations, must enthrone the policy in order to add the "status authority" to the "competence authority" it should already possess. The policy must also contain its own action plan, a procedure for moving the organisation from status quo to the status the policy describes, and it must provide an explicit procedure for regularly evaluating and, if necessary, remodelling the policy over time. This is especially important if the envisioned state of affairs deviates considerably from the status quo. When the surveys at Catholic Hospital show that the majority of the staff are reluctant to grant patients the right to refuse blood transfusions, the OE working group should include in its recommendation for a "treat without blood" policy a number of steps to facilitate implementing the policy. The steps could include a programme for educating staff, assigning staff of certain rank to coordinate the process for patients who refuse blood, or encouraging conscientious objectors to make themselves known.

If the practice that the policy regulates is not carefully implemented and evaluated the situation risks combining the flaws of individual decision making (people are not prepared to evaluate each case on its own) with those of having a policy (people will rely on a coordinating procedure that does not really work).

\section{CONCLUSION}

I argue that the adequate response of a HCO to moral disagreement is a process of consensus building that leads to hospital wide policies, because such a process better respects the autonomy of all parties involved than individual decision making, and better ensures the quality, fairness, and efficiency of the decision making process.

Policy writing for morally controversial medical practices differs from policy writing for standard medical practices, because it resembles more closely a political process than an authoritarian directive on standard medical care. Such a process therefore has to meet the five additional requirements discussed above, which are primarily procedural, in order to claim legitimate authority over the actions of individual members of the organisation. The task of policy writing must be as concerned with the process of reaching consensus by open deliberation as with the actual outcome of the process. This is to say that different solutions can be equally reasonable, depending on how much weight the deliberative process gives to any of the substantive principles that reflect the obligations of a HCO: care for the sick; treat your employees with respect; act in AQ A public spirit, and use resources reasonably.

\section{ACKNOWLEDGEMENTS}

Many thanks for helpful comments and discussion of this material to Dennis F Thompson, Anne Mills, Sandra Badin, K Robin Pierce, and members of the Faculty Seminar of the Center for Ethics and the
Professions at Harvard University. I am also most grateful to James Sabin, Judy Johnson, and John Emans for providing me with the opportunity to observe the work of their organisational ethics programmes in practice and to Jean McVeigh for her generous support in editing the latest drafts of this paper.

Funding: this work has been generously funded by the Edmond J Safra foundation.

\section{REFERENCES}

1 Buchanan A. Toward a theory of the ethics of bureaucratic organisations. Bus Ethics Q 1996;6:419-40.

2 Trevino LK, Butterfield KD, McCabe DL. The ethical context in organisations: influences on employee attitudes and behaviors. Bus Ethics $Q$ 1998;8:447-76.

3 Cropanzano R. Deontic justice: the role of moral principles in workplace fairness. J Org Behavior 2003;24:1019-24.

4 Thompson DF. The institutional turn in professional ethics. Ethics Behav 1999;9:109-18.

5 Vinicky JK, Smith ML, Connors Jr RB, et al. The Jehovah's Witness and blood: new perspectives on an old dilemma. J Clin Ethics 1990;1:65-74.

6 Weir RF, Peters C. Affirming the decisions adolescents make about life and death. Hastings Cent Rep 1997;27:29-40.

7 Tait AR, Voepel-Lewis T, Malviya S. Do they understand? (part II): assent of children participating in clinical anesthesia and surgery research. Anesthesiology 2003;98:609-14

8 Scott ES, Reppucci ND, Woolard JL. Evaluating adolescent decision making in legal contexts. Law Hum Behav 1995;19:221-44.

9 Beauchamp TL, Childress JF. Principles of biomedical ethics. New York: Oxford University Press, 2001:57-272.

10 Benn SI. Persons and values: reasons in conflict and moral disagreement. Ethics 1984;95:20-37.

11 Sunstein C. Why societies need dissent. Cambridge, MA: Harvard University Press, 2003:16.

12 Schein E. Culture: the missing concept in organisation studies. Adm Sc Q 1996;41:109-19.

13 Schaver F. Playing by the rules: a philosophical examination of rule based decision making in law and in life. New York: Oxford University Press, 1993:136.

14 French PA. Collective responsibility and the practice of medicine. J Med Philos 1982;7:65-85.

15 Walzer M. Obligations: essays on disobedience, war, and citizenship. Cambridge, MA: Harvard University Press, 1970:28.

16 Raz J. Authority and justification. Authority. New York: New York University Press, 1990:134.

17 Gutmann A, Thompson DF. Democracy and disagreement: why moral conflict cannot be avoided in politics, and what should be done about it. Cambridge, MA: Harvard University Press, 1996:41, 50.

18 McMahon C. Authority and democracy: a general theory of government and management. Princeton, NJ: Princeton University Press, 1994:12.

19 Habermas J. Reconciliation through the public use of reason: remarks on John Rawls's political liberalism. J Philos 1995;92:109-31.

20 Clouser KD, Gert B. Morality versus principlism. In: Gillon R, Lloyd A, eds. Principles of health care ethics. New York: John Wiley and Sons, 1994:251.

21 Winkler EC, Gruen R. First principles: substantive ethics for health care organisations. J Healthc Manag 2005 [In press.]

22 Daniels N, Sabin J. The ethics of accountability in managed care reform. Health Aff (Millwood) 1998;17:50-64.

23 Buchanan A. Trust in managed care organisations. Kennedy Inst Ethics J 2000;10:189-212.

24 Hirschman AO. Exit, voice, and loyalty: response to declines in firms, organisations, and states. Cambridge, MA: Harvard University Press, 1970:33.

25 McGee G, Caplan AL, Spanogle JP, et al. A national study of ethics committees. Am J Bioeth 2001;1:60-4.

26 Pentz RD. Beyond case consultation: an expanded model for organisational ethics. J Clin Ethics 1999;10:34-41. 